\title{
Material and Characteristics Analysis of a Carbon Fiber Badminton Shuttle by Using a Wind Tunnel
}

\author{
Jih-Tseng Yeh ${ }^{1}$ and Wei-Jen Chen ${ }^{2 *}$
${ }^{1}$ Mechanical \& Engineering Integration Department, CTCI Advanced Systems Inc., 5F, No. 52, Sec.3, Nangang Road, Taipei 11510, Taiwan, R.O.C. ${ }^{2}$ Department of Aeronautical Engineering, Chaoyang University of Technology 168, Jifeng E. Rd., Wufeng District, Taichung 413310, Taiwan, R.O.C.

(Received December 29, 2020; accepted April 26, 2021)

Keywords: carbon fiber shuttle, wind tunnel, wind resistance

Badminton has long been a popular sport for all ages. However, owing to the high wearing rate of shuttles, this sport is not considered to be environmentally friendly. To mitigate this problem and improve the durability of shuttles, many manufacturers have been researching and developing synthetic shuttles. Different from most of the synthetic shuttles with plastic skirts, the one produced by VICTOR is made of a carbon fiber and foam material for the shaft and the vane of the feathers. The aim of this study is to determine the characteristics of such a carbon fiber shuttle. Because the flying speed of a shuttle is the fastest among all ball-type sports, the aerodynamic performance of shuttles has mainly been considered in academic studies. A wind tunnel is one of the best ways to evaluate the aerodynamic characteristics of a shuttle. For this reason, in addition to inviting players for actual badminton games and using other tools or instruments for measuring, testing, and comparison purposes, we used a small custom-made wind tunnel with an airspeed sensor unit and tension measurement meter to collect data from natural feather shuttles and synthetic shuttles, especially from carbon fiber shuttles. The main purpose of this paper is to introduce the implementation of a wind tunnel to evaluate different types of shuttles.

\section{Introduction}

A traditional shuttle is made with a cork head and goose or duck feathers. ${ }^{(1)}$ Because of their short lifetime, badminton players must spend a considerable amount of money on shuttles. Therefore, many manufacturers have been eagerly developing shuttles of different materials other than duck and goose feathers, hoping to increase the durability of shuttles while retaining their performance.

Since Carlton Sports introduced the world's first synthetic shuttle in the 1950s, many synthetic shuttles with different materials or designs have been developed. ${ }^{(2-4)}$ Despite their better durability, the performance of most synthetic shuttles was inferior to those made of natural feathers. Therefore, professional players and most mid-level and high-level nonprofessional players still prefer shuttles made of natural materials.

*Corresponding author: e-mail: dancelighten@gmail.com

https://doi.org/10.18494/SAM.2021.3270 
However, the acquisition of natural shuttle feathers is easily impacted by different causes such as avian influenza, so the output of the product is relatively unstable. In addition, the population of badminton players has been increasing. These reasons have caused the price of shuttles to rise. Therefore, shuttle manufacturers are still attempting to develop synthetic shuttles. It is hoped that the overall performance of synthetic shuttles can be improved so that they can replace natural ones to solve the problems of acquisition difficulty, low durability, and high price.

Before carbon fiber shuttles were launched on the market, some defects were found in synthetic shuttles with a plastic feather skirt, such as an unstable flight trajectory and higher flying speed when smashed. Further verification is therefore needed to establish whether shuttles made with a carbon fiber and foam material can meet the requirements and expectations of badminton players. ${ }^{(5)}$

\section{Research Method and Process}

\subsection{Research methods and results other than wind tunnel testing}

The objects of comparison in this experiment are natural shuttles and carbon fiber shuttles. In addition to wind tunnel testing, the other research methods and results are as follows.

\subsubsection{Basic data measurement and comparison}

The basic data of two types of badminton shuttles were measured in accordance with documents of the Badminton World Federation (BWF) Equipment Certification Programme, Shuttlecock. ${ }^{(6)}$ After measurement and comparison, it was found that the carbon fiber shuttle had a similar appearance to the natural one, and the various other data were similar. The weights of the shuttle and the appearance and size of each vane were slightly different, but the values were all within $\pm 10 \%$ tolerance, thus conforming to the BWF specifications, and the measured weights were within $\pm 0.10 \mathrm{~g}( \pm 2 \%)$. Although the head sizes of the shuttles were the same, the weight may vary due to the density of the cork and the amount of glue used to fix the cotton rope. The weight of glue was about $1 \mathrm{~g}$, which was one-fifth of the weight of a shuttle.

By comparing top views of the carbon fiber and natural shuttles, it was found that their crosssectional areas were slightly different. In Fig. 1, the red area shows the natural shuttle projection and the blue area was the carbon fiber shuttle projection. The pixels of the shuttle projections on the two images indicated that the size difference was 3.18\%. This gave the carbon fiber shuttle a smaller area on the windward side, thus reducing its wind resistance.

\subsubsection{Questionnaire survey}

Two rounds of 21-point games were arranged for different groups. After these games, players were asked to evaluate the carbon fiber shuttle, and all the shuttles were examined for their 


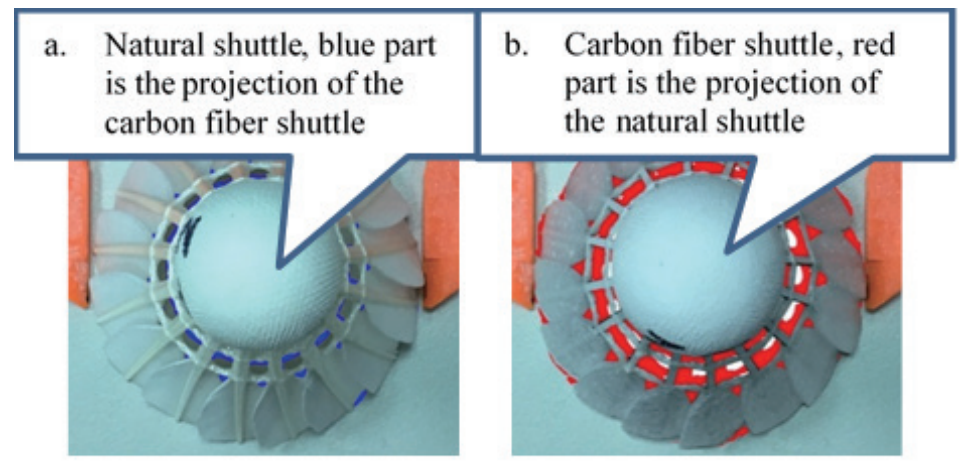

Fig. 1. (Color online) Comparison between natural and carbon fiber shuttles.

appearance and damage. Extra shuttles were also provided to other players for two weeks to experience the new type of shuttle, after which they filled in a questionnaire.

The players of the first group were high-level nonprofessional players. After the end of the first game, they reported that the speed of the shuttle increased during the game. Visual inspection of the shuttle showed that the foam material at the end of the vane had begun to peel off. Moreover, the carbon fiber shaft and the second loop of the cotton rope used to fix the shaft had clearly become softened.

The second group consisted of leisure players who had played badminton for more than five years. Although they thought that the head of the carbon fiber shuttle was slightly heavier, they were satisfied with the performance of the carbon fiber shuttle compared with the natural one. Visual inspection after the game showed that many shafts of the carbon fiber feather had broken. This may have been caused by an improper hitting position so that the shafts had collided with the racket frame. The peeling of the foam material at the end of the vane was relatively slight compared with the shuttles used by the first group, for which the flying speed was much higher. In the questionnaire given to the other players, the durability of the carbon fiber shuttle received the highest rating and the price received the lowest rating (Table 1).

\subsubsection{Structural observation}

We used a scanning electron microscope (SEM) to compare the structural differences between the natural and carbon fiber shafts and analyze the damage to the broken shafts taken from the used shuttles.

From the SEM images of the broken natural shaft, the broken carbon fiber shaft, and the delaminated carbon fibers obtained with different magnifications, it was found that the outer part of the fractured natural shaft had no trace of tension fracture. This explained why the dry and brittle shaft broke instantly after being directly hit by the racket.

The cross section of the broken carbon fiber shaft was similar to that of the natural shaft. The broken matrix and reinforcement of the composite could be seen on the cross section. Instead of bending, the laminated carbon fiber also broke directly after being hit by a racket, as shown in Fig. 2. 
Table 1

Players' evaluation of shuttles.

\begin{tabular}{lccccccc}
\hline Group & Appearance & Net shot & Deep shot & Smash & Cut & Durability & Price \\
\hline 1st & 9 & 7.75 & 7 & 4.75 & 8.25 & 4.75 & 5.5 \\
2nd & 9 & 7.25 & 8 & 7 & 8 & 7.75 & 7 \\
Questionnaire & 7.93 & 7.52 & 7.59 & 7.41 & 7.59 & 8.30 & 7.33 \\
\hline
\end{tabular}

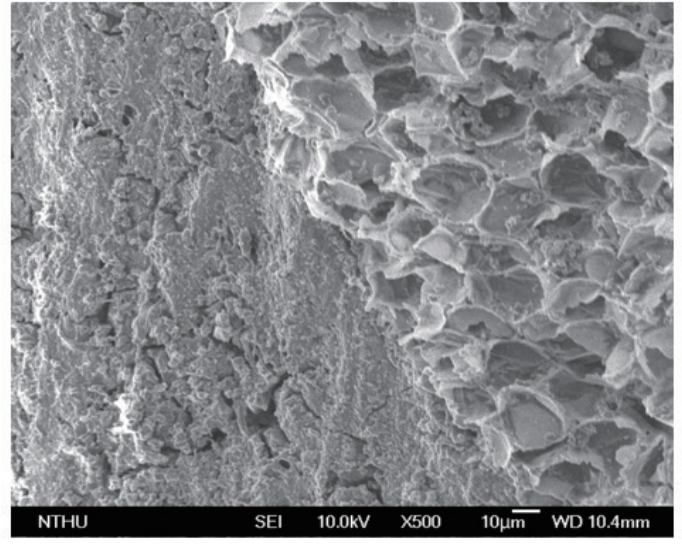

(a)

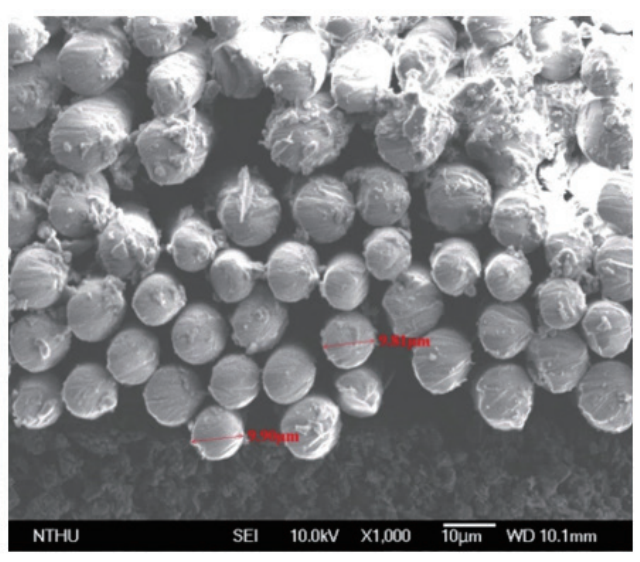

(b)

Fig. 2. (Color online) Fractured cross sections of (a) natural feather and (b) carbon fiber shafts.

Regarding the delamination of the carbon fiber shaft, it was found from the SEM image that hardly any of the matrix of the reinforcement material remained on the surface in the delaminated area. This means that the adhesion between the reinforcement material and the matrix was poor, and cracks and breaks were also found on the carbon fiber shaft, as shown in Fig. 3.

In the case of good adhesion, even after being damaged by external forces, there should still be a large amount of the matrix attached to the surface of the carbon fiber. After being hit by the racket or constantly bent by wind pressure, this poor adhesion of the carbon fiber shaft made it easy for delamination to occur, then the deformation of the skirt increased, further reducing the resistance to delamination.

\subsubsection{Experimental shuttle}

On the basis of the above findings, the experimental shuttle in Fig. 4 was made of the foam material and carbon fiber. To offset the weight of the increased foam material, this shuttle used a smaller carbon fiber shaft. The reduced bending resistance of the shaft due to its smaller size was complemented by the mutual traction between the foamed vanes. The plan to improve the shuttle was as follows:

a. To prevent the shaft from easily being broken by the racket frame, an elastic material can be used to cover the carbon fiber shaft. However, owing to the weight limitation of a shuttle, in addition to absorbing part of the impact force from the racket, the material must be extremely lightweight, such as a foam material. 


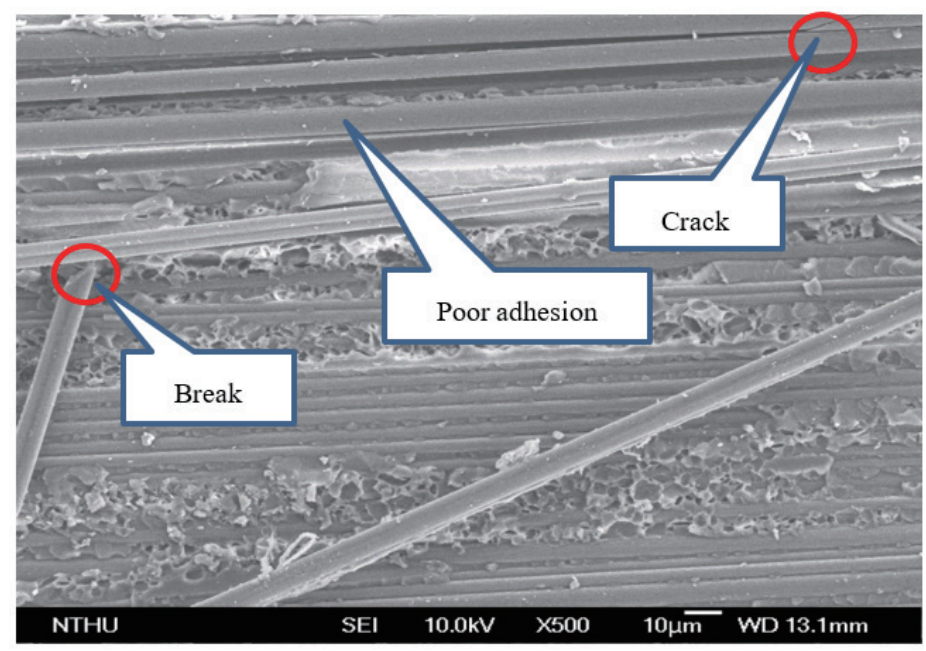

Fig. 3. (Color online) Delaminated area of the carbon fiber shaft.
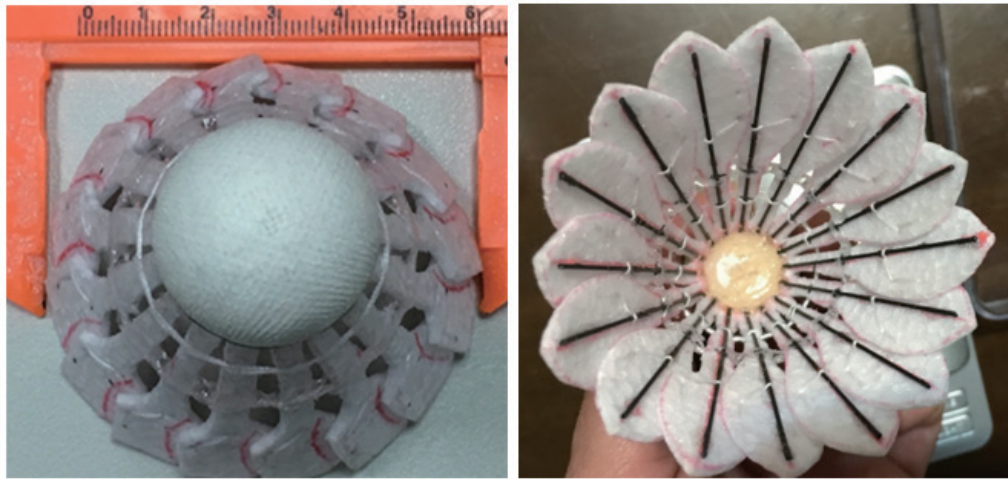

Fig. 4. (Color online) Custom-made experimental shuttle.

b. Because the carbon fiber shaft was thinner, the frontal wind-resistance area of the carbon fiber shuttle was smaller, and the wind resistance would be poorer than that of the natural shuttle under a high wind speed. To fix this problem, the material covering the shaft was placed on the outside of the shaft to increase the wind-resistance area of the shuttle.

\subsection{Wind tunnel test}

The main method used in this study was wind tunnel testing. This was performed using a custom-made wind tunnel to compare the wind resistance of different shuttles under different airspeeds, the deformation of various shuttle feather skirts under high airspeeds, and the spin wobbling of shuttles. Plastic shuttles and the custom-made shuttle were tested in this phase to provide extra reference data. 


\subsubsection{Introduction to wind tunnels}

There are many types of wind tunnels used for different fields of research. These include tunnels with supersonic and subsonic wind speeds; those with a suction fan, blowing fan, or compressed air to provide the testing airflow; open- and closed-loop wind tunnels; and so on. The main structure of a wind tunnel is usually divided into the airpower section, airflow rectification section, contraction section, and test section. The data required for this study was used to compare different types of badminton shuttles, so a simplified custom-made wind tunnel was used.

\subsubsection{Components of the custom-made wind tunnel}

The simple custom-made wind tunnel consists of several parts as shown in Fig. 5.

a. Electric power supply unit

The electric ducted fan motor is driven by two $30 \mathrm{C}, 22.2 \mathrm{~V}$ DC lithium batteries through a 120 A electronic transmission unit. A $12 \mathrm{~V}$ DC transformer provides the power supply for the cooling fans and electronic transmission controller.

b. Airflow generation unit

A high-speed airflow is provided by a $105 \mathrm{~mm}$ electric ducted fan.

c. Control unit

The fan speed can be adjusted via a control knob to change the output of electronic transmission, thus providing a certain speed $(0-60 \mathrm{~m} / \mathrm{s})$ of the airflow.

d. Airspeed sensor unit

The airspeed of the wind tunnel is provided through a PIXHAWK pitot tube and a digital differential pressure sensor, then is sent to a PIXHAWK flight controller and presented by Mission Planner Software on a notebook computer. ${ }^{(7,8)}$ Except for the software and the notebook, these items are usually used for a fixed-wing RC aircraft.

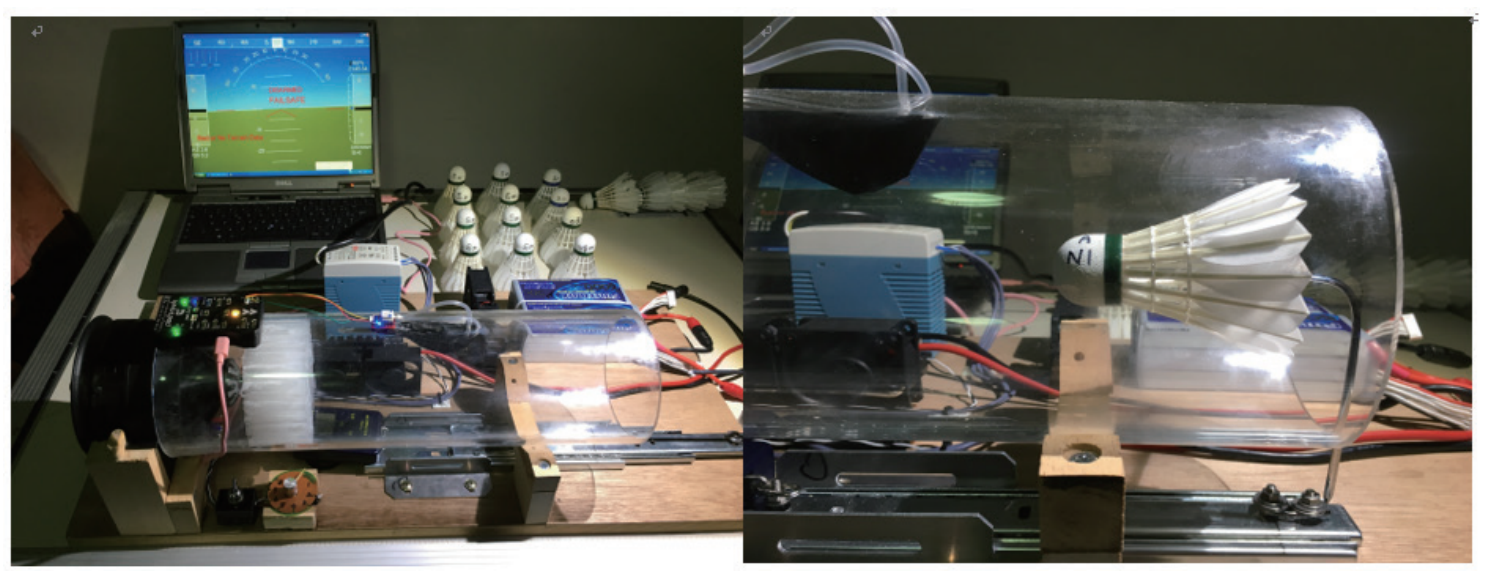

Fig. 5. (Color online) Custom-made wind tunnel. 


\section{e. Cooling system}

The electric ducted fan is cooled by its own airflow. Four additional cooling fans are installed to cool the electronic transmission unit and the lithium batteries.

\section{f. Airflow duct}

The main airflow used to test the wind resistance and skirt deformation of a shuttle is provided by the electric fan via the horizontal air duct with simple rectification. An additional L-type adaptor and vertical air duct are used to provide the vertical airflow to monitor the spin wobbling of a shuttle.

g. Shuttle test unit

A rod is fixed on a slide rail connected to a tension meter. A shuttle to be tested can be installed on the rod to obtain the wind resistance at different airspeeds. The deformation of the shuttle skirt at a high speed can also be observed from the top of the transparent air duct.

\subsubsection{Wind tunnel test procedure}

a. Preliminary step

- The shuttles were divided into different groups. The groups were denoted as AN (brand-new natural shuttles), BN (brand-new carbon fiber shuttles), AU (used natural shuttles), BU (used carbon fiber shuttles), CN (brand-new plastic skirt shuttles), and HM (custom-made carbon fiber shuttles).

- The used shuttles were obtained by having the high-level players perform $10 \mathrm{~min}$ of deep shots from both ends of the court using each brand-new natural and carbon fiber shuttle.

- Both the front and rear areas of the wind tunnel were cleared to prevent the fan from being damaged by foreign objects so as not to affect the airflow at the outlet of the wind tunnel.

b. Wind-resistance test

- The shuttles to be tested were sequentially placed on the rod in the test area before setting the airspeed for the test.

- The maximum airspeed of the custom-made wind tunnel was $60 \mathrm{~m} / \mathrm{s}(216 \mathrm{~km} / \mathrm{h})$. To obtain stable results, the range of the airspeed used in the tests was set between $25 \mathrm{~m} / \mathrm{s}(90 \mathrm{~km} / \mathrm{h})$ and $55 \mathrm{~m} / \mathrm{s}(198 \mathrm{~km} / \mathrm{h})$.

- The wind-resistance values were read out from the tension meter at different airspeeds.

- The steps above were repeated 10 times for each shuttle to obtain average values and standard deviations.

c. Deformation test

- A measuring ruler was installed behind the shuttle to be tested, then the control knob was adjusted to the maximum test airspeed $(55 \mathrm{~m} / \mathrm{s})$ to observe the maximum deformation of the high-pressure wind-resistance zone at the end of the shuttle, i.e., the tips of the feathers.

d. Spin wobbling observation

- An L-type adaptor and a vertical air duct were installed and the fan speed was adjusted to obtain a suitable airflow. Then, the shuttle to be tested was placed on the top of the air duct outlet to observe the spin wobbling of the shuttle. 


\subsubsection{Results and comparison of the tests}

a. Wind-resistance comparison

The test results of each shuttle group showed that when the airspeed was below $35 \mathrm{~m} / \mathrm{s}$, the resistance of all shuttles was roughly the same. The difference in resistance between each shuttle group began to increase from $35 \mathrm{~m} / \mathrm{s}$. The values of the resistance of each group at different speeds are shown in Fig. 6. Overall, the resistance of the carbon fiber shuttle was lower than that of the natural one. In particular, in the BU group (used carbon fiber shuttles), the resistance under high airspeeds was significantly reduced. This may explain why the high-level players felt the shuttle speed increased. The performance of the brand-new group $\mathrm{CN}$ shuttles was similar to that of the group BU shuttles. That is also why most of the advanced players did not like using the shuttles with plastic skirts. For the custom-made shuttles (HM), since the deformation of the skirt seemed limited by the extra loop of cotton rope, the wind resistance was much higher than that of the BU and $\mathrm{CN}$ shuttles and close that of the natural shuttle.

b. Comparison of feather skirt deformation

The results of the skirt deformation tests at the airspeed of $55 \mathrm{~m} / \mathrm{s}$ showed that the deformation of the BU group carbon fiber shuttle was $6.06 \%$ higher than that of the new shuttle. This difference resulted in the reduction in wind resistance mentioned above.

The deformation of the group $\mathrm{CN}$ shuttles was greater than that of the group BU shuttles. This explained why the wind resistance was much lower at high speeds. The deformation of the HM shuttle was close to that of the natural one and only $3 \mathrm{~mm}$ of skirt deformation was found. These results indicate that the greater the deformation of the shuttle, the lower its wind resistance (see Table 2 and Fig. 7).

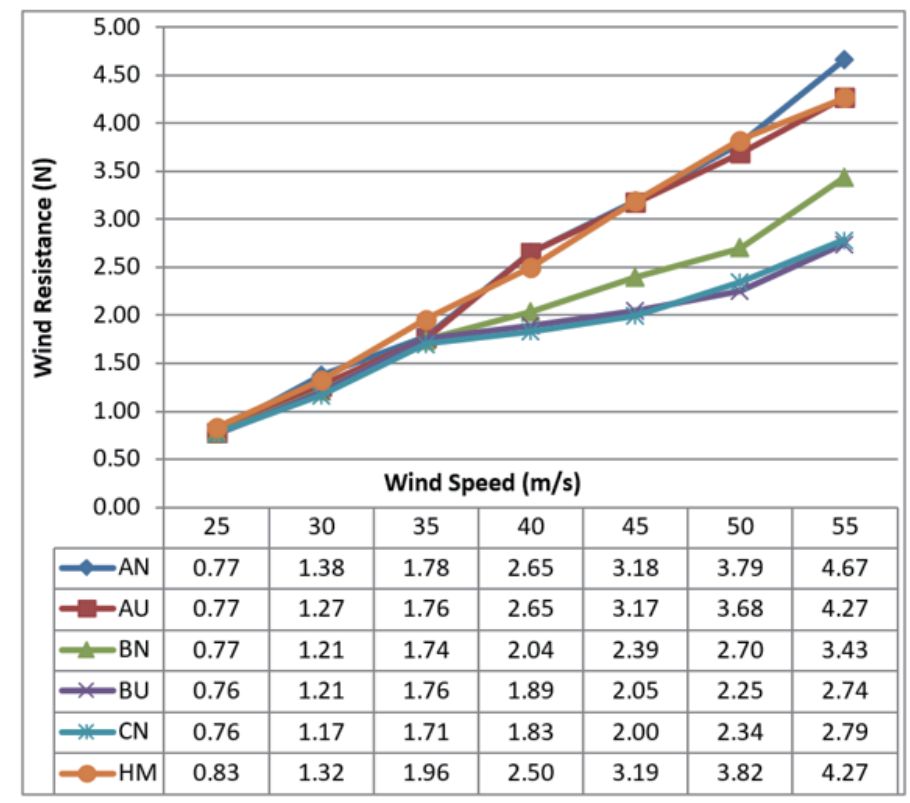

Fig. 6. (Color online) Wind resistance of shuttles. 
Table 2

Feather skirt deformation at airspeed of $55 \mathrm{~m} / \mathrm{s}$.

\begin{tabular}{lccc}
\hline Group & Skirt diameter & Deformation at $55 \mathrm{~m} / \mathrm{s}$ & Difference between new and used \\
\hline AN & $66 \mathrm{~mm}$ & $3 \mathrm{~mm}, 4.55 \%$ & - \\
AU & $66 \mathrm{~mm}$ & $4 \mathrm{~mm}, 6.06 \%$ & $+2.01 \%$ \\
BN & $66 \mathrm{~mm}$ & $4 \mathrm{~mm}, 6.06 \%$ & - \\
BU & $66 \mathrm{~mm}$ & $8 \mathrm{~mm}, 12.12 \%$ & $+6.06 \%$ \\
CN & $64 \mathrm{~mm}$ & $11 \mathrm{~mm}, 17.19 \%$ & - \\
HM & $64 \mathrm{~mm}$ & $3 \mathrm{~mm}, 4.69 \%$ & - \\
\hline
\end{tabular}

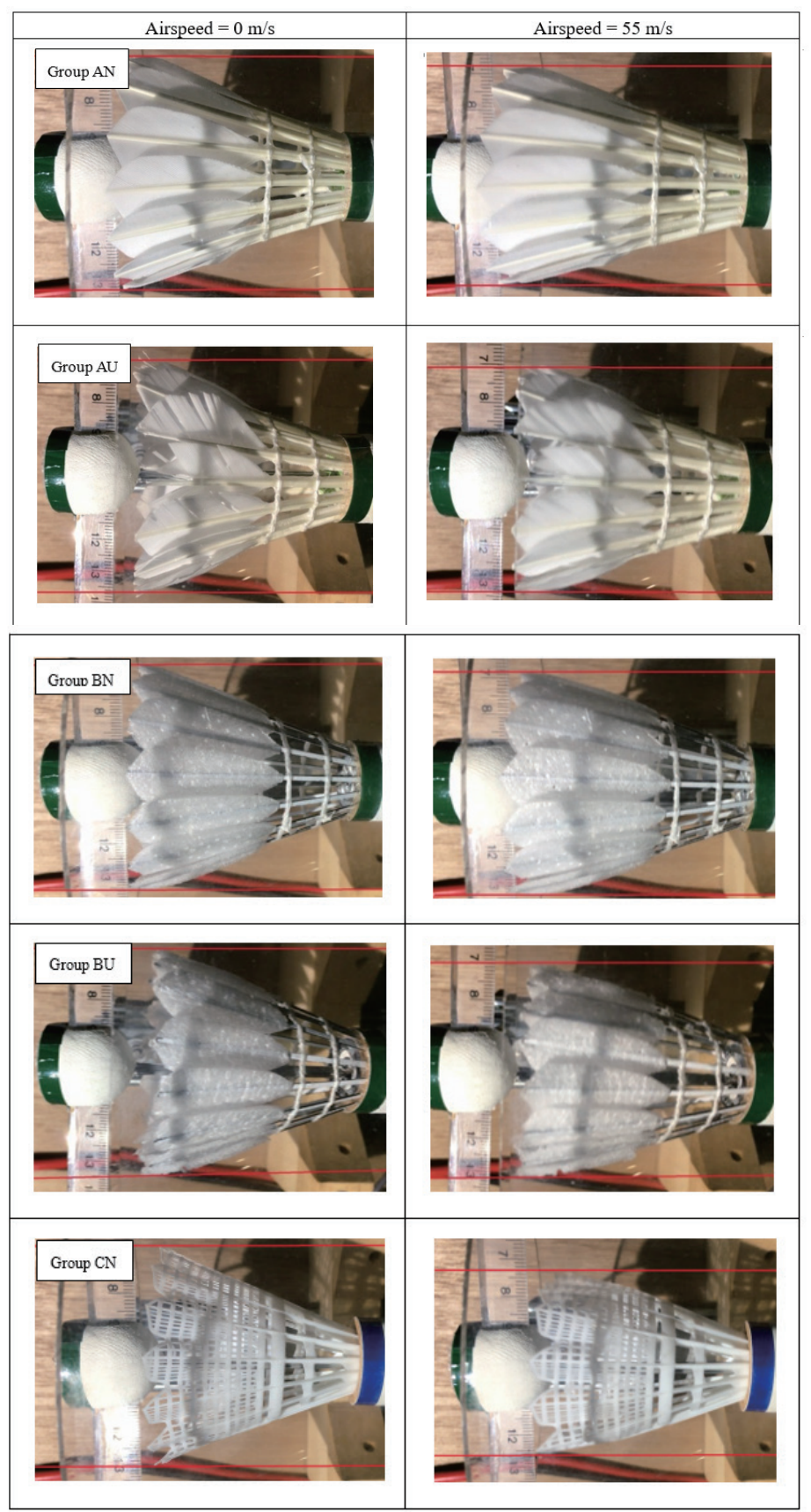

Fig. 7. (Color online) Feather skirt deformation at airspeed of $55 \mathrm{~m} / \mathrm{s}$. 


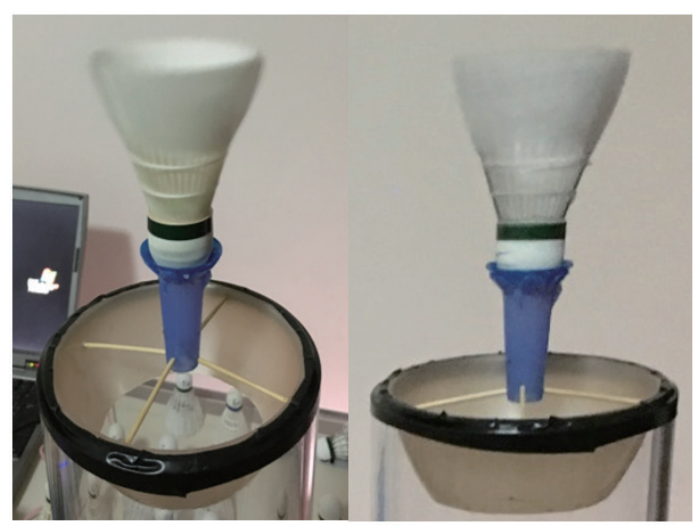

Fig. 8. (Color online) Observation of wobbling during shuttle spin.

c. Spin wobbling observation and comparison

By observing the spin of the different shuttles, it was found that they all spun when subjected to a certain wind force. This rotation improves the stability of the shuttle's flight. The test showed that all the new shuttles spun stably, as shown in Fig. 8. However, because the angle of attack of each feather was not exactly the same on the natural shuttle, slight wobbling occurred during the spinning process. The degree of this wobbling is also used as a basis to classify the stability of a shuttle in the BWF documentation, with an obvious imbalance occurring for a shuttle with a broken feather.

\section{Conclusions}

In addition to the basic data measurement and comparison among natural and carbon fiber shuttles, the tests in this study mainly explored the resistance distribution of shuttles and the deformation of the feather skirt, where a simple wind tunnel, SEM, and other measuring tools were implemented to directly obtain results.

By comparing the natural and carbon fiber shuttles, we found that their appearances were similar, as well as their weights, sizes, and other data. The results of a questionnaire survey and feedback from players using both types of shuttles showed that the players were satisfied with the appearance of the carbon fiber shuttles.

However, from the wind tunnel tests, we found that the wind resistance of the carbon fiber shuttles was lower than that of the natural shuttles under a high wind speed, especially for used shuttles. This was a fatal defect of carbon fiber shuttles for high-level players because the flying speed of the shuttle increased when the wind resistance was lower. The deformation of the shuttle skirt was the cause of the lower resistance. The wind resistance, or drag, of an object is mainly calculated by using the reference area of the object, which is usually fixed. ${ }^{(9)}$ When the feather skirt of a shuttle is not deformed, the aerodynamic resistance of the shuttle can be calculated from its orthographic projection area. However, because the end of the feather skirt is deformed under the influence of wind pressure at high speeds, its drag coefficient is the same as the Reynolds number. The Reynolds number changes with the wind speed. ${ }^{(10-13)}$ In addition, 
when the shuttle travels in the air, part of the airflow also flows to the rear through the vane gap. The spin of the shuttle during the high-speed flight will also affect the wind resistance. ${ }^{(14)}$

Regarding the limitations of the tests in this study, because there are seven main factors affecting the overall performance of a shuttle, i.e., its weight, weight distribution, center of gravity, support strength, frontal wind resistance, wind resistance around the axis, and resistance against hitting, although we have evaluated the effects of the resistance and support strength, other evaluations such as the effects of the center of gravity and the resistance induced by the airflow through the shuttle shaft gap will require the use of more accurate wind tunnels, experimental instruments, or simulation software to perform more tests and obtain more precise results.

In the past, the best test method for newly designed shuttles was wind tunnel tests, which can directly give the changes in the wind resistance of shuttles. Nowadays, using powerful computers, it is also possible to simulate the performance of shuttles by creating models and using computational fluid dynamics simulation software. ${ }^{(15)}$ From the viewpoint of experimental testing methods, powerful computers and various simulation software are helpful to individuals, research institutes, and manufacturers to develop a new generation of products by improving the efficiency and reducing the cost of R\&D.

We are currently in an era of continuous innovation in material science and applications. After plastic feather skirts, carbon fiber and foamed vanes should be just another step. In the future, if the material selection and design can be effectively improved, the shortcomings of synthetic shuttles such as poorer global performance, unstable flight trajectory, and higher flying speed when smashed may be overcome. Ecologically decomposable and lower-cost materials with acceptable performance for most professional and amateur badminton players may also be developed.

\section{References}

1 Shuttlecock, Wikipedia: https://en.wikipedia.org/wiki/Shuttlecock (accessed March 2018).

2 Espacenet - patent search website: https://worldwide.espacenet.com/advancedSearch?locale=en_EP (accessed March 2018).

3 Y. Satoshi, O. Masao, and T. Yutaka: European Patent Application, Application No.: 10809857.5, from MIZUNO Corporation, Japan (June, 2012).

4 Feather vs. Nylon, Badminton Shuttles: https://yumo.ca/blogs/badminton-information/badminton-shuttlesfeather-vs-nylon (accessed March 2018).

5 S. J. Chen, T. W. Wang, H. C. Wang, and C. Y. Chang: Publication of patent application, TW Patent Number: 105204910, VICTOR RACKETS INDUSTRIAL CORP' (1 September 2016).

6 BWF Equipment Certification Programme, Shuttlecock: http://system.bwf.website/docume-nts/folder_1_81/ folder_1_140/folder_1_165/1\%20Shuttlecocks\%20Overview.pdf (accessed March 2018).

7 Pitot tube, Wikipedia: https://en.wikipedia.org/wiki/Pitot tube (accessed March 2018).

8 Air Density Calculator: https:/www.omnicalculator.com/physics/air-density (accessed March 2018).

9 Drag Coefficient, Wikipedia: https://en.wikipedia.org/wiki/Drag coefficient (accessed March 2018).

10 B. D. Texiera, C. Cohena, D. Quéréa, and C. Claneta: Shuttlecock Dynamics, 9th Conf. International Sports Engineering Association (ISEA, 5 March 2012).

11 A. J. Cooke: The Aerodynamics and Mechanics of Shuttlecocks, Ph.D. Thesis (Cambridge University, Cambridge, UK, 1992).

12 F. Alam, H. Chowdhury, C. Theppadungporn, and A. Subic: A Study of Badminton Shuttlecock Aerodynamics, School of Aerospace, Mechanical and Manufacturing Engineering, RMIT University, ICME09-FM-17 (26-28 December, 2009). 
13 Y. B. Wen: The Impact of Air Resistance on the Badminton Flight Path and Velocity, Master's thesis, Department and Graduate Institute of Physics, NCUE TAIWAN (July, 2013).

14 S. Kittaa, H. Hasegawaa, M. Murakamib, and S. Obayashic: Aerodynamic Properties of a Shuttlecock with Spin at High Reynolds Number, 5th Asia-Pacific Congr. Sports Technology (APCST, 15 May 2011).

15 J. Hart: Simulation and Understanding of the Aerodynamic Characteristics of a Badminton Shuttle, The 2014 Conf. International Sports Engineering Association (2014).

\section{About the Authors}

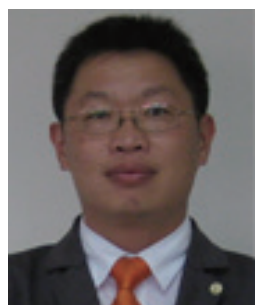

Jih-Tseng Yeh received his B.S. degree from China University of Science and Technology, Taiwan, in 2006 and his M.S. degree from China University of Science and Technology, Taiwan, in 2018. From 2007 to 2011, he was a highspeed train driver for THSRC, Taiwan. Since 2012, he has been a flight simulator field engineer at Hsinchu AFB, Taiwan. (sonny.wdns@gmail.com)

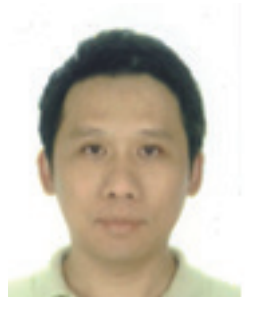

Wei-Jen Chen received his M.S. degree from Chung Yuan Christian University, Taiwan, in 2004 and his Ph.D. degree from National Tsing Hua University, Taiwan, in 2010. He worked in the electronics industry from 2010 to 2014. From 2015 to 2019, he was an assistant professor at China University of Science and Technology, Taiwan. Since 2019, he has been an assistant professor at Chaoyang University of Technology. His research interests are in nanocomposites, fuel cells, and composite material manufacturing processes and testing. (t2018092@cyut.edu.tw) 